\title{
Gene expression patterns of novel visual and non-visual opsin families in immature and mature Japanese eel males
}

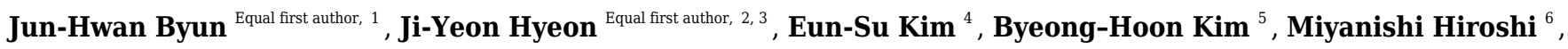 \\ Kagawa Hirohiko $^{6}$, Yuki Takeuchi $^{7}$, Se-Jae Kim ${ }^{3}$, Akihiro Takemura ${ }^{7}$, Sung-Pyo Hur ${ }^{\text {Corresp. } 2,3}$ \\ ${ }^{1}$ Graduate School of Engineering and Science, University of the Ryukyus, Nishihara, Okinawa, Japan \\ 2 Jeju Research Institute, Korea Institute of Ocean Science \& Technology, Jeju, Jeju, Republic of Korea \\ 3 Department of Biology, Jeju National University, Jeju, Jeju, Republic of Korea \\ 4 Jejudo marine fish-culture cooperative, Jeju, Jeju, Republic of Kora \\ 5 Marine Science Institute, Jeju National University, Jeju, Jeju, Republic of Korea \\ 6 Department of Marine Biology and Environmental Sciences, Faculty of Agriculture, University of Miyazaki, Gakuen-Kibanadai-Nishi, Miyazaki, Japan \\ 7 Department of Chemistry, Biology and Marine Science, Faculty of Science, University of the Ryukyus, Nishihara, Okinawa, Japan \\ Corresponding Author: Sung-Pyo Hur \\ Email address: hursp@jejunu.ac.kr
}

This study was carried out to identify and estimate physiological function of a new type of opsin subfamily present in the retina and whole brain tissues of Japanese eel using RNA-Seq transcriptome method. A total of 18 opsin subfamilies were identified through RNA-seq. The visual opsin family included Rh2, SWS2, FWO, DSO, and Exo-Rhod. The non-visual opsin family included four types of melanopsin subfamily (Opn4x1, Opn4x2, Opn4m1, and Opn4m2), peropsin, two types of neuropsin subfamily (Opn5-like, Opn5), Opn3, three types of TMT opsin subfamily (TMT1, 2, 3), VA-opsin, and parapinopsin. In terms of changes in photoreceptor gene expression in the retina of sexually mature and immature male eels, DSO mRNA increased in the maturation group. Analysis of expression of opsin family gene in male eel brain before and after maturation revealed that DSO and SWS2 expression in terms of visual opsin mRNA increased in the sexually mature group. In terms of non-visual opsin mRNA, parapinopsin mRNA increased whereas that of TMT2 decreased in the fore-brain of the sexually mature group. The mRNA for parapinopsin increased in the mid-brain of the sexually mature group, whereas those of TMT1 and TMT3 increased in the hind-brain of the sexually mature group. DSO mRNA also increased in the retina after sexual maturation, and DSO and SWS2 mRNA increased in whole brain part, suggesting that DSO and SWS2 are closely related to sexual maturation. 


\section{Gene expression patterns of novel visual and non-visual opsin families in \\ 2 immature and mature Japanese eel males}

3

4 Jun-Hwan Byun ${ }^{*}$, Ji-Yeon Hyeon ${ }^{2,3 *}$, Eun-Su Kim, Byeong-Hoon Kim ${ }^{5}$, Miyanishi Hiroshi ${ }^{6}$,

5 Kagawa Hirohiko ${ }^{6}$, Yuki Takeuchi ${ }^{7}$, Se-Jae Kim ${ }^{3}$, Akihiro Takemura ${ }^{7}$ and Sung-Pyo Hur ${ }^{2,3}$

6

$7 \quad{ }^{1}$ Graduate School of Engineering and Science, University of the Ryukyus, 1 Senbaru, Nishihara,

8 Okinawa 903-0213, Japan

9 2Jeju Research Institute, Korea Institute of Ocean Science \& Technology, Jeju, Jeju, Republic of 10 Korea

$11{ }^{3}$ Department of Biology, Jeju Natireonal University, 102 Jejudaehak-ro, Jeju 63243, Republic of

12 Korea

13 4Jejudo marine fish-culture cooperative, Jeju, Jeju, Republic of Korea

$14{ }^{5}$ Marine Science Institute, Jeju National University, Jeju, Jeju, Republic of Korea

$15{ }^{6}$ Department of Marine Biology and Environmental Sciences, Faculty of Agriculture, University

16 of Miyazaki, Gakuen-Kibanadai-Nishi, Miyazaki, Japan

$17{ }^{7}$ Department of Chemistry, Biology and Marine Science, Faculty of Science, University of the

18 Ryukyus, Nishihara, Okinawa, Japan

19

20 Short title: opsin mRNA expression in Japanese eel

$21 \quad======$

22 Corresponding author

23 Sung-Pyo Hur 
24 Department of Biology, Jeju National University, 102 Jejudaehak-ro, Jeju, 63243, Republic of 25 Korea

26

27 E-mail: hursp@jejunu.ac.kr

28

* These authors contributed equally to this work.

30

\section{Abstract}

This study was carried out to identify and estimate physiological function of a new type of opsin subfamily present in the retina and whole brain tissues of Japanese eel using RNA-Seq transcriptome method. A total of 18 opsin subfamilies were identified through RNA-seq. The visual opsin family included Rh2, SWS2, FWO, DSO, and Exo-Rhod. The non-visual opsin family included four types of melanopsin subfamily (Opn4x1, Opn4x2, Opn4m1, and Opn4m2), peropsin, two types of neuropsin subfamily (Opn5-like, Opn5), Opn3, three types of TMT opsin subfamily (TMT1, 2, 3), VA-opsin, and parapinopsin. In terms of changes in photoreceptor gene expression in the retina of sexually mature and immature male eels, DSO mRNA increased in the maturation group. Analysis of expression of opsin family gene in male eel brain before and after maturation revealed that DSO and SWS2 expression in terms of visual opsin mRNA increased in

42 the sexually mature group. In terms of non-visual opsin mRNA, parapinopsin mRNA increased 43 whereas that of TMT2 decreased in the fore-brain of the sexually mature group. The mRNA for 44 parapinopsin increased in the mid-brain of the sexually mature group, whereas those of TMT1 45 and TMT3 increased in the hind-brain of the sexually mature group. DSO mRNA also increased 
46 in the retina after sexual maturation, and DSO and SWS2 mRNA increased in whole brain part,

47 suggesting that DSO and SWS2 are closely related to sexual maturation.

48

49

50

51

52

53

54

55

56

57

58

59

60

61

62

63

64

Keywords: opsin, photoreceptor, Japanese eel, Anguilla japonica, sex maturation

50

1

\section{Introduction}

4 Living organisms recognize various environmental information (light, water temperature, salinity, etc.) according to their specific ecology and have ecologically evolved based on the information. Organisms perceive the external environment through light, transmit the information to the brain, synchronize the biological clock operation to activate the metabolism, and control the physiological and ecological functions by inducing the secretion of endocrine hormones such as melatonin (Benoit, 1978; Falcon, 1999, 2007; Campbell et al., 2001). Mammals have two types of photoreceptor proteins (rhodopsin, cone-opsin) that perceive light in the retina of the eye, and different types of photoreceptors recognize the signals of light (wavelength of light, intensity of light, direction of light, and periodicity) (Hestings and Maywood, 2000; Tada et al., 2009). A photoreceptor is a visual sensory cell capable of recognizing light of a specific wavelength. Photoreceptors also refer to an opsin protein receptor that actually absorbs light and converts it into chemical energy. Vertebrate photoreceptors are regulated by opsin, a superfamily of G-protein-coupled receptor (GPCR), opsin, with an inverse agonist 11-cis retinal chromophore, covalently bound. Indeed, retinal molecules selectively absorb various spectrum of light depending on the formation of binding with the opsin protein (reviewed in Pugh and Lamb, 2000). Absorption of light at a specific wavelength leads to 
70 conversion into all-trans form that binds the opsin and transducing proteins, thereby activating a

71 series of visual sensitive-related cellular signal transduction processes (Terakita, 2005). Opsin

72 superfamily is broadly divided into visual and non-visual opsins. There have been extensive

73 studies on vision function of opsin-based photopigment. However, when the opsin was found in

74 tissues such as avian pineal and amphibian skin, opsins were unofficially divided into visual and

75 non-visual groups (Okano et al., 1994; Kojima and Fukada, 1999; Van Gelder, 2001). As extra-

76 ocular tissues cannot form images, this classification was suggested. Visual opsin initiates the

77 visual transduction cascade, whereas non-visual opsin is involved in circadian entrainment

78 (Doyle et al., 2008) and retinal metabolism (Bellingham et al., 2003).

79 The habitats of marine organisms, especially fish, vary in depth and region, ranging from

80 freshwater to brackish areas. The light conditions of these habitats are different in terms of 81 turbidity, color, and brightness (Bowmaker, 1994, 2008). For example, in the case of deep-sea

82 snailfish inhabiting relatively deep-water areas, the spectral sensitivities of the rod and cone

83 photoreceptors react to the blue light (Sakata et al., 2015). In contrast, in the case of black bream,

84 shallow-sea fish, cone photoreceptors (Rh2 or MWS), have maximal light absorbance

85 wavelength $(\lambda \max )$ at 545 to $575 \mathrm{~nm}$, which is the dominant light in their habitat (Shand et al.,

86 2002). Thus, it is presumed that animals have obtained a unique visual system that have made

87 them adapt to the light environment of their habitats in the process of evolution.

88 The Japanese eel, Anguilla japonica, has been known to have a dynamic life cycle. It migrates

89 to the seawater areas during spawning season and spawns. Sexually immature eels are yellow

90 whereas sexually mature ones are silver. The leptocephalus undergo metamorphosis into glass

91 eels while moving through ocean currents, migrate to freshwater areas where they spend most

92 the time in their life cycle (Tsukamoto, 1992; Tatsukawa, 2003). Thus, the Japanese eel 
93 experiences diverse changes in water environment during its life cycle, which differs greatly

94 from other fish and animals.

95 To date, photoreceptor studies on Anguillid have identified fresh water rhodopsin (FWO)

96 (Zhang et al., 2000), deep-sea rhodopsin (DSO) (Zhang et al., 2000), Rh1d (European eel, $A$.

97 anguilla and Japanese eel, A. japonica and giant mottled eel, A. marmorata) (Wang et al., 2014),

98 Rh2 (European eel and giant mottled eel) (Cottrill et al., 2009), and SWS2 (European eel and 99 giant mottled eel) (Wang et al., 2014). Molecular biological studies on photo sensitivities of 100 these visual pigments and studies on the expression mechanism of photoreceptors according to 101 ecological stages (glass eel, yellow eel, and silver eel) have been actively conducted. However, 102 the presence or function of a subfamily other than the above-mentioned four types of visual 103 opsin or non-visual opsin subfamily in Anguilla species has not been reported yet. Physiological 104 studies of photoreceptors in vertebrate animals have reported that pinopsin and VA-opsin 105 (Okano et al., 1994; Soni and Foster, 1997) in the brain of birds and exo-rhodopsin in the pineal 106 gland of zebrafish directly affect body color change and reproductive physiology (Kojima et al., 107 2000; Collin et al., 2009). Thus, it is considered that other types of photoreceptors, except for the 108 previously reported opsins, may play an important reproductive physiological role in Japanese 109 eel but there has been no further investigation into it.

110 In this study, we investigated the opsin subfamily present in the retina and whole brain tissues 111 of Japanese eel inhabiting Northeast Asia using the RNA-Seq transcriptome. In addition, we 112 examined the opsin subfamily mRNA levels in sexually immature and mature eels using qPCR 113 method. These results identify the physiological role of photoreceptors in the maturation process 114 of Japanese eels and, thus, can be used as a basic material for studies on photoreceptor 115 mechanisms including the effect of environmental factors on maturation and visual adaptation. 
117 Materials \& Methods

118 Experimental fish

119 We purchased Japanese eels, A.japonica, inhabiting brackish water at Hadori, Gujwaeup, Jejusi, 120 Jeju, South Korea in September at 2016. The wild fish were kept in Lava seawater center in Jeju 121 Techno-park, Jeju, South Korea $\left(33^{\circ} \mathrm{N}, 126^{\circ} \mathrm{E}\right)$. The fish were reared for 1 weeks in acryl tank 122 (800L/capacity) with recirculation system (natural photoperiod= approximately 12L:12D, water 123 temperature $20 \pm 1^{\circ} \mathrm{C}$ ). For the study of the maturation induction of the Japanese eel, males were 124 purchased from an eel aquafarm (Hanwool aquafarm, Gwangju, South Korea). The obtained eels 125 were acclimated in the freshwater round acrylic tank (1 ton/capacity) for at least one week. Light 126 conditions were maintained at 12L:12D using fluorescent bulbs (10W, 600 lx, PPFD= 10.0 $127 \mu \mathrm{molm}^{-2} \mathrm{~s}^{-1}, \lambda \mathrm{p}=545 \mathrm{~nm}$ ) light on at 06:00 and light off at 18:00), and the temperature of the 128 water was maintained at $20 \pm 1^{\circ} \mathrm{C}$. All experiments were conducted in compliance with the 129 guidelines of Institutional Animal Care and Experimental Committee of the Jeju National 130 University. The protocol was approved by the Animal care and use committee of the Jeju 131 National University (No. 2016-0039).

133 For the retina and whole brain RNA-transcriptome analysis (Fig. 1), wild caught Japanese 134 eels (body weight: 233-726 g and body length: 55.3-80.7 cm) were reared for 1 weeks in acryl 135 tank. For the sampling of experimental fish, the retina and the brain were isolated from Japanese eels at 12:00h and 24:00h ( $\mathrm{n}=12$, six females and six males) after anesthesia with tricaine

137 methanesulfonate (MS-222, Sigma-Aldrich, ST., USA). The collected tissues were frozen using 138 liquid nitrogen and stored at $-80{ }^{\circ} \mathrm{C}$ until used for analysis. 
For maturation artificially induction of Japanese eels, only males (initial body weight: 186.1

$140227.1 \mathrm{~g}, \mathrm{n}=6$ ) were selected and reared in the freshwater tank for at least one week. Later, the

141 water was replaced with sea water for one week, and the fish were reared for eight weeks and

142 intraperitoneally injected with human chorionic gonadotropin $\left(\mathrm{n}=6, \mathrm{hCG}, 1 \mathrm{IU} / \mathrm{g}^{-1}\right)$ dissolved in

143 saline $(150 \mathrm{mM} \mathrm{NaCl})$ at one-week intervals for sexually maturation. During the maturation

144 induction, photoperiod of 12L: 12D (lights on $=07: 00$, lights off $=19: 00)$ and water temperature

145 of $20 \pm 1^{\circ} \mathrm{C}$ were maintained, and a complete recirculating aquaculture system (800L/capacity).

146 Fluorescent bulbs (20W, approximately $600 \mathrm{~lx}, 10.0 \mu \mathrm{molm}^{-2} \mathrm{~s}^{-1}$ at $545 \mathrm{~nm}$ ) were situated above

147 on the tank to provide an illuminance at water surface of 600 lx. After eight weeks of

148 intraperitoneal injection, maturation was determined by the presence or absence of spermiation

149 and histological observation of the testis. For analysis of opsin family genes mRNA level

150 changes in the retina and brain part of Japanese eels before and after maturation, the brain was

151 dissected into the fore-brain, mid-brain, and hind-brain (Fig. 2). The extracted tissues were

152 frozen using liquid nitrogen and stored at $-80^{\circ} \mathrm{C}$ until used for analysis.

153

154 Total RNA isolation and cDNA synthesis

155 Total RNA was isolated from the retina and three parts of the brain (fore-, mid-, and hind-)

156 using RNA-iso plus (Takara-Bio, Otsu, Japan) according to the manufacturer`s protocol. After

157 isolated the total RNA, quality, and amount-checked on a 2100 bioanalyzer RNA 6000 NANO

158 chip (Bio-Rad, Hercules, CA, USA) and electrophoresis. cDNA was synthesized using the

159 Transcriptor High Fidelity cDNA Synthesis kit (Roche-diagnostics, Indianapolis, IN, USA) by

160 following the manufacturer`s protocol.

162 cDNA library construction and massively parallel sequencing 
163 RNA-Seq paired end libraries were prepared using the Illumina TruSeq RNA Sample

164 Preparation Kit v2 (catalog \#RS-122-2001, Illumina, San Diego, CA). Total RNA was isolated

165 from the retina and brain, respectively. After removal of genomic DNA contamination, RNA

166 quality and quantity were assessed by 2100 bioanalyzer RNA 6000 NANO chip (Bio-Rad). High

167 quality total RNA extracted from retina and brain of X individuals were then pooled,

168 respectively. Starting with total RNA, mRNA purified using poly (A) selection was chemically

169 fragmented and converted into single-stranded cDNA using random hexamer priming. Next, the

170 second strand is generated to create double-stranded cDNA. Library construction begins with

171 generation of blunt-end cDNA fragments from ds-cDNA. Then A-base added to the blunt-end

172 in order to make them ready for ligation of sequencing adapters. After the size selection of

173 ligates, the ligated cDNA fragments which contain adapter sequences are enhanced via PCR

174 using adapter specific primers. The library was quantified with KAPA library quantification kit

175 (Kapa biosystems KK4854) following the manufacturer's instructions. Each library is loaded on

176 Illumina Hiseq2000 platform, and we performed high-throughput sequencing (read length

$1772 \times 100)$ to ensure that each sample meets the desired average sequencing depth.

179 Preprocessing and de novo reconstruction of transcriptome,

180 The bases from 5' end and 3'end of each read with low quality and adapter sequences were 181 trimmed using Trimmomatic (ver. 0.3.6, Bolger, Lohse \& Usadel, 2014), then low averaged 182 quality $(\mathrm{Q}<25)$ were removed by PRINSEQ lite (ver. 0.20.4, Schmieder \& Edwards, 2011).

183 Cleaned raw reads from retina and brain RNA were pooled, then mapped to the Anguilla 184 japonica draft genome sequence (Ref) using tophat2 (ver.2.1.0, Kim, 2013), then de novo 185 transcriptome reconstruction was performed by a genome-guided Trinity (ver. 2.3.2, Grabherr et 
186 al., 2011) with bam mapping result. To remove redundant contigs and create an unigene set, the 187 assembled contigs were clustered and filtered using cd-hit-est with default parameters (CD-HIT 188 package, Li \& Godzik, 2006).

189

190

191

192

193 194

195

196

197

198

199

200

201

202

203

204

205

206

207 208

\section{Analysis of the opsin DNA sequence}

To search opsin family genes from the assembled transcriptome sequences of Japanese eel, the tBlastn program was utilized (E-value $<0.01)$ on zebrafish opsin protein sequences as queries. The ORF regions of Japanese eel opsin candidates were found through ORF Finder (http://www.ncbi.nlm.nih.gov/gorf/gorf.html), then presumed protein sequences were aligned with teleost opsin family proteins. A phylogenetic tree was constructed by the maximum-likelihood algorithm using RAxML (Stamatakis, 2014). For quantifying the identified opsin family genes expressions, cleaned reads were mapped on reconstructed contigs by Bowtie2 (Langmead \& Salzberg, 2012), then the expression levels were estimated using Tigar2 (Nariai et al., 2014).

\section{Quantitative real-time RT-PCR (qPCR)}

Real-time qPCR reactions were performed using the Dice real time thermal cycler (TaKaRaBio) and SYBR Premix Ex Taq ${ }^{\mathrm{TM}}$ II (TaKaRa-Bio). Gene specific primers used for qPCR were designed using Primer3 plus (Primer Biosoft) and are provided in Table S1. Each PCR reaction mix contained $50 \%$ of SYBR Premix, $0.2 \mu \mathrm{M}$ of each forward and reverse primer, and $2 \mu \mathrm{l}$ of diluted cDNA template by nuclease-free water. The initial 1 min denaturation was followed by 40 cycles of denaturation for $5 \mathrm{~s}$ at $95^{\circ} \mathrm{C}$, annealing and extension for $1 \mathrm{~min}$ at $60{ }^{\circ} \mathrm{C}$. To ensure the specificity of the PCR amplicons, the temperature of the sample was gradually raised from 60 to $95^{\circ} \mathrm{C}$ as the last step of the PCR reaction and a melting curve analyzed. The primers were 
209 successfully tested in the different cDNA samples of the Japanese eel, evaluating that each

210 primer should amplify a single product, reflected as a single peak in the melting curve analysis.

211 The relative mRNA expression levels of target genes were calculated using the ${ }^{\Delta \Delta} \mathrm{Ct}$ method, and

212 the reference gene was virtually defined as the average of the threshold cycles $(\mathrm{Ct})$ for EF1 $\alpha$.

213

\section{Histological analysis}

215 The eel testis was fixed in Bouin's fluid. Fixed testis samples were dehydrated through an 216 ethanol series, embedded in paraffin wax, and sectioned to 7-8 $\mu \mathrm{m}$ thickness. Sectioned tissues

217 were stained with Mayer's hematoxylin and eosin. State of the sexual maturation was classified 218 into the following 2 stages: immature stage (spermatogonia and spermatocyte; Fig. 3A) and 219 maturation stage (fully spermatozoa; Fig. 3B).

220

\section{Statistics}

222 All statistical analyses were performed using GraphPad Prism 8.0.2 Software. Comparisons of 223 opsin genes expression levels between sexually immature and mature group were performed by the Unparied t test. In the present study, $P<0.05$ was accepted as statistically significant.

225

226

\section{Results}

227

228 229 230 231

\section{RNA-Seq transcriptome analysis}

Total RNA extracted from the whole brain and retinal tissues of Japanese eels were analyzed using the NGS method. After the adapter trimming and quality filtering, 150,898,925 paired-end reads were survived and used for de novo transcriptome reconstruction. As a result of cd-hit-est clustering, a total of 313,671 contigs $(\mathrm{N} 50=965)$ were obtained. tBlastn and phylogenetic 
232 analysis revealed that a total of 18 opsin subfamilies were identified in the retina and the whole

233 brain through RNA-seq (Fig. 4). Among them, the visual opsin families of Japanese eels

234 included rhodopsin2 or middle wave sensitive pigment (Rh2 or MWS), short wavelength-

235 sensitive opsin 2 or blue light sensitive opsin (SWS2), fresh water rhodopsion (FWO), deep-sea

236 water rhodopsin (DSO), and exo-rhodopsion (Exo-Rhod). The non-visual opsin families

237 included four types of melanopsin subfamily (Opn4x1, Opn4x2, Opn4m1, and Opn4m2),

238 peropsin, two types of neuropsin (Opn5-like, Opn5), Opn3 (encephalopsin), three types of

239 teleost multiple tissue opsin (TMT1, TMT2, and TMT3), VA-opsin (vertebrate ancient opsin)

240 and parapinopsin.

241

\section{Changes of GSI}

243 Sexually immature and mature eels were classified based on the histological observation of testis

244 before and after hCG treatment. In the beginning, spermatogonia was mostly observed in the 245 testis of eel males. After eight weeks of hCG injection, spermiation was found in most of male

246 eels, and spermatozoa was mostly observed in lobules. The gonadosomatic index (GSI) was 0.20

$247 \pm 0.01$ at the beginning and was $25.7 \pm 1.4$ after maturation, showing a significant difference $(P$

$248>0.0001$, Fig. 3C)

Changes in opsin family gene expression in the retina between sexually immature and

251

252

253

254

mature eels

Eighteen opsin families identified using the RNA-Seq method were divided into visual opsin (Fig. 5) and non-visual opsin (Fig. 6) families. Then, the mRNA abundance in the retina of sexually immature and mature eels was analyzed using qPCR. In terms of visual opsin 
255 expression, the mRNA abundance of DSO increased in the sexually mature group (Fig. 5B),

256 whereas those of FWO and Rh2 were low in the sexually mature group (Fig. 5C, D). Non-visual 257 opsin mRNA showed no significant difference between sexually immature and mature groups 258 (Fig. 6).

259

\section{Changes in opsin family gene expression in brain of sexually immature and mature eels}

The brains of the sexually immature and mature eels were dissected into the fore-brain, midbrain, and hind-brain. Then, the opsin families that showed significant differences were investigated as in the retina $(P<0.05)$. In terms of visual opsin expression in the brain, DSO and SWS2 mRNA abundance increased in the fore-brain, mid-brain, and hind-brain of the mature group. Other visual opsin mRNAs did not show significant differences in whole brain part (Fig.

7). In terms of non-visual opsin expression in the brain, mRNA abundance of parapinopsin and low in the mature group (Fig. 9G). Opn4m2 and parapinopsin mRNA abundance increased in the mid-brain (Fig. 8K, 9Q). TMT1 and TMT3 mRNA abundance increased in the hind-brain of the 270 mature group (Fig. 9F, L).

271

\section{Discussion}

RNA-Seq transcriptome analysis was performed to examine 18 photoreceptor genes in the retina

274 and whole brain of Japanese eels. As a result, two types of cone opsin (SWS2, Rh2) were

275 identified. However, the presence of a long wavelength-sensitive pigment (LWS) in the long

276 wavelength region was not confirmed in this study. In general, organisms must have at least two

277 cone opsin with different spectra to distinguish colors. Species with one type of cone opsin are 
278 considered as "monochromatic vision" or color-blind (Bowmaker et al., 1994). Eels have two or

279 more cone opsin, so they can recognize colors. However, they recognize the wavelength of the

280 narrower region compared with other animals or other fish species. A study suggested that the

281 European eel has two types of cone opsin subfamily, Rh2 (or MWS) and SWS2 cones, so it can

282 distinguish colors (Cottrill et al., 2009). However, the giant mottled eel showed only one type of

283 cone cell that detected a limited range of the optical spectrum ( $\lambda \max )$ of $500 \mathrm{~nm}$ to $535 \mathrm{~nm}$

284 (Wang et al., 2014). Japanese eels are genetically and ecologically similar European eels; thus, it

285 is presumed that they can recognize colors through two types of cone opsin. In addition,

286 Japanese eels are nocturnal fish and have evolved in a way that they have adapted to dark habitat

287 and/or nocturnal habits. Thus, it is considered that their photoreceptors recognizing the light

288 spectrum of the long wavelength band region may have been functionally atrophied or

289 photoreceptor may have not existed. Similarly, species living in deep-sea or those evolved to

290 adapt to dark environments have been reported to have fewer cone opsins (Mas-Riera, 1991;

291 Pankhurst and Conroy, 1987). SWS1 and LWS gene expression levels were higher in fresh water

292 fish than those in fish inhabiting seawater (Lin et al., 2017). This is because in most freshwater

293 environments, most of the ultraviolet rays penetrating the water surface can be recognized by

294 organisms because of low water depth, while the fish living in deep-sea (50m or more) tend to

295 lose the LWS gene because the long wavelength (red) is not transmitted to deep-sea regions (Lin 296 et al., 2017).

297 Japanese eel, which was investigated in this study, live in a shallow freshwater region during 298 most of their life span except for spawning. This eel will migrate to deep-sea area of at least 100 299 m (Cottrill et al., 2009; Tsukamoto, 1992). Regarding this, they may share some genetic

300 characteristics with the deep-sea fish and in this study cone opsin was predicted to be one of the 
301 possible genes. The distribution and physiological function of cone opsin appear to be different 302 depending on the level of ecological evolution. In Anguillid sp., only four types opsins (DSO, 303 FWO, Rh2 and SWS2) (Cottrill et al., 2009; Zhang et al., 2000) were studied. Therefore, it is 304 necessary to carry out additional molecular biological and biochemical studies on the range of 305 recognition of color in eels. In this study, opsin families were identified through RNA-seq 306 method, and then highly expressed genes in the retina and brain were analyzed by qPCR. As a 307 result, Parapinopsin mRNA was predominantly expressed in the whole brain, but peropsin and 308 Opn5 were relatively highly expressed in the retina. According to previous studies on opsin 309 expression, the expression of rhodopsin genes in the retina and brain in the ayu (Masuda et al., 310 2003; Minamoto and Shimize, 2003), Atlantic salmon (Philp et al., 2000), Japanese eel (Zhang et 311 al., 2000), and percomorph fishes (Cortesi et al., 2015), showed different photoreceptor types 312 and expression sites. However, only the limited physiological function of opsin has been 313 reported.

314 Non-visual opsin was named in the 1990s, and it has been known to affect circadian rhythms 315 in mammals, reproduction in birds, light avoidance in amphibian larvae, and neural development 316 during egg development in fish (Beaudry et al., 2017). A study on non-visual opsin showed

317 Opn4 expression in the retinal ganglion in mammals, but Opn4 gene was expressed in the retina, 318 brain and skin in non-mammals (Belingham et al., 2006). In addition, VA-opsin is known to be 319 expressed in the hypothalamus and gonads in birds and fish, and it directly stimulates GnRH in 320 the hypothalamus by recognizing wavelength changes due to photoperiod changes (Davies et al., 321 2010; Grone et al., 2007). TMT opsin is expressed in most tissues and embryos in the case of 322 zebrafish. In particular, TMT opsin is expressed in cell lines associated with light entrain able 323 clock (Moutsaki et al., 2003). 
In this study, DSO expression increased, and FWO, Opn4m2, VA-opsin, SWS2 and Rh2

325

326

327

328

329

330

331

332

333

334

335

336

337

338

339

340

341

342

343

344

345

346

expression decreased in the retina during sexual maturation. All of the three brain areas showed the increased DSO and SWS2 expressions. Consistent with the results of this study, a previous study on the photoreceptors of the Japanese eel, reported that DSO expression increased and FWO expression decreased in silver eels (Zhang et al., 2000). In the case of European eel, DSO expression also increased to make eels adapt to the environment before the spawning migration in the early sexual maturation stage. In the late sexual maturation stage, European eels enter deep-sea beyond $100 \mathrm{~m}$ depth to spawn, thereby showing a decrease in FWO expression (Zhang et al., 2000). In addition, Japanese conger eel changes its habitat environment from fresh water to open sea while moving from juvenile stage to sexual maturation. To adapt to the changed environment, FWO was mainly expressed in the retina during the juvenile stage, and then DSO expression started to be increased during sexual maturation (Zhang et al., 2002). This may have resulted from Japanese conger eel's adaptation to the environmental change related to light in the process of migrating to the spawning ground. Analysis of opsin family gene mRNA levels in the Japanese eel brain before and after maturation showed that DSO and SWS2 expressions increased after maturation in all three areas of brain. In addition, DSO expression increased in the retina after maturation, suggesting that DSO is closely related to maturation. However, it is unclear whether the increase in DSO and SWS2 expressions in the brain affects maturation.

In recent years, there have been some studies on the reproductive physiological function of VA-opsin belonging to the non-visual opsin (deep brain photoreceptor) family in the brain. VA opsin was cloned from Atlantic salmon (Soni and Foster, 1997), and VA-long (VAL-opsin) was discovered in zebra fish (Kojima et al., 2000). Immunohistochemistry studies on Atlantic salmon have reported the existence of opsin-like protein in the hypothalamic nucleus magnocellularis 
347 preopticus, suggesting its potential gonadal development control function. There have been few

348 studies on non-visual opsin in the brain, especially its relevance to gonadal development.

349 However, photoperiod action did not influence the gonadal development in ayu without both

350 eyes and pineal gland (Suzuki, 1975), and opsin immune-positive fibers passing through basal

351 hypothalamus were observed in the hypothalamus of Atlantic salmon (Philp et al., 2000). These

352 results suggest that the opsin present in the brain directly affects gonadal development. The level

353 of expression of GnRH located at the top of the BPG axis directly affects reproduction. It is

354 unclear as to whether VA-opsin regulates the expression level of GnRH in the hypothalamus of

355 Japanese quail, but VA-opsin, which affected GnRH expression, was identified in GnRH cells

356 (Garcia-Fernadez et al., 2015). Other opsin subfamilies other than VA-opsin may also affect the

357 reproduction system in the hypothalamus, but more research is needed to investigate this

358 hypothesis.

359

360 Conclusions

361 Thousands of opsins have been identified and are divided into eight groups (Terakita, 2005; Yau

362 and Hardie, 2009; Peirson et al., 2009; Terakita et al., 2012). The current data set shows the

363 diversity of opsins in the animal kingdom because the whole genome sequence is determined in

364 many animals. However, there has been a lack of information on the physiological functions

365 other than the molecular structure or biochemical signals in the retina. In particular, the study of

366 ecologically unique species such as Japanese eels is considered as very important in terms of

367 evolution. In this study, 18 types of opsins were identified in the brain and retina of Japanese eels

368 of which 14 types were new opsin genes. Expression of opsins mRNA in the brain and retina was

369 variable; SWS2 expression was high in all areas of the brain of the sexually mature eels, and 
370 TMT3 expression significantly increased in hind-brain. These results suggest that SWS-related

371 shortwave region is directly related to the maturation of Japanese eels. However, follow-up

372 studies are required to demonstrate the relevance. Japanese eels have very unique ecological

373 characteristics, as mentioned above. Unlike other fish species, eco-physiological studies on

374 Japanese eels are necessary to induce artificial maturation through environmental control (light,

375 water temperature etc.), and various studies on the photosensitivity should be continuously

376 carried out.

377

378 Acknowledgements

379 The authors are thankful to Chulhong Oh, Moonjeong Lee, Soojin Heo, Dohyung Kang of the

380 Korea Institute of Ocean Science and Technology (KIOST) for their expert assistance and

381 helpful suggestions.

382

383 Additional Information and Declarations

384 Funding

385 This work was supported by a grant from the National Institute of Fisheries Science (R2019003) and 386 supported by the project 'Innovative marine production technology driven by LED-ICT convergence 387 photo-biology', Ministry of Oceans and Fisheries, Korea, and by a grant from the National Institute of 388 Fisheries Science (R2017038).

389

390 Competing Interests

391 The authors declare there are no competing interests.

392

393 Author Contributions 
394 Jun-Hwan Byun, Ji-Yeon Hyeon conceived and designed the experiments, performed the

395 experiments, analyzed the data, prepared figures and/or tables, authored or reviewed drafts of the 396 paper, approved the final draft.

397 Eun-Su Kim and Yuki Takeuchi conceived and designed the experiments, performed the 398 experiments, authored or reviewed drafts of the paper, approved the final draft.

399 Byeong-Hoon Kim and Miyanishi Hiroshi analyzed the data, contributed 400 reagents/materials/analysis tools.

401 Kagawa Hirohiko, Se-Jae Kim and Akihiro Takemura conceived and designed the experiments, 402 analyzed the data, authored or reviewed drafts of the paper, approved the final draft.

403 Sung-Pyo Hur conceived and designed the experiments, analyzed the data, prepared figures 404 and/or tables, authored or reviewed drafts of the paper, approved the final draft. 405

406 Data Availability

407 The following information was supplied regarding data availability:

408 The raw sequencing reads are publicly available in the Sequence Read Archive (SRA) of the 409 GenBank database under the accession numbers DRR194268 to DRR194271.

\section{REFERENCES}

413 Altschul SF, Gish W, Miller W, Myers EW, Lipman DJ. 1990. Basic local alignment search 414 tool. Journal of molecular biology 215(3):403-410.

415 Beaudry FEG, Iwanicki TW, Mariluz BRZ, Darnet S, Brinkmann H, Schneider P, Taylor

416 JS. 2017. The non-visual opsins: eighteen in the ancestor of vertebrates, astonishing increase 
417

418

419

420

421

422

423

424

425

426

427

428

429

430

431

432

433

434

435

436

437

438

in ray-finned fish, and loss in amniotes. Journal of Experimental Zoology Part B: Molecular and Developmental Evolution 328(7): 685-696.

Bellingham J, Chaurasia SS, Melyan Z, Liu C, Cameron MA, Tarttelin EE, Iuvone PM, Hankins MW, Tosini G, Lucas RJ. 2006. Evolution of melanopsin photoreceptors: discovery and characterization of a new melanopsin in nonmammalian vertebrates. PLoS biology 4(8), e254.

Bellingham J, Tarttelin EE, Foster RG, Wells DJ. 2003. Structure and evolution of the Teleost extraretinal rod-like opsin (errlo) and ocular rod opsin (rho) genes: Is teleost rho a retrogene?. Journal of Experimental Zoology Part B: Molecular and Developmental Evolution 297(1), 1-10.

Benoit JM. 1978. Chronobiologic study in the domestic duck. II. Physiological mechanism of the chronobiologic action of visible light on the gonads of the male duck. Chronobiologia 5:158-168.

Bolger AM, Lohse M, Usadel B. 2014. Trimmomatic: A flexible trimmer for Illumina sequence data. Bioinformatics 30:2114-2120. DOI: 10.1093/bioinformatics/btu170.

Bowmaker JK, Govardovskii VI, Shukolyukov SA, Zueva LV, Hunt DM, Sideleva VG, Smirnova OG. 1994. Visual pigments and the photic environment: The Cottoid fish of Lake Baikal. Vision research 34(5), 591-605

Bowmaker JK. 2008. Evolution of vertebrate visual pigments. Vision research 48(20):20222041.

Campbell SS, Murphy PJ, Suhner AG. 2001. Extraocular phototransduction and circadian timing systems in vertebrates. Chronobiology international 18:137-172. 
439 Chen C, Khaleel SS, Huang H, Wu CH. 2014. Software for pre-processing Illumina next-

440 generation sequencing short read sequences. Source code for biology and medicine 9(1), 8.

441 Collin SP, Davies WL, Hart NS, Hunt DM. 2009. The evolution of early vertebrate

442 photoreceptors. Philosophical Transactions of the Royal Society B: Biological

$443 \quad$ Sciences 364(1531): 2925-2940.

444 Cortesi F, Musilová Z, Stieb SM, Hart NS, Siebeck UE, Malmstrøm M, Tørresen OK,

445 Jentoft S, Cheney KL, Marshall NJ, Carleton KL, Salzbuger W. 2015. Ancestral

446 duplications and highly dynamic opsin gene evolution in percomorph fishes. Proceedings of

447 the National Academy of Sciences 112(5): 1493-1498.

448 Cottrill PB, Davies WL, Semo M, Bowmaker JK, Hunt DM, Jeffery G. 2009. Developmental

449 dynamics of cone photoreceptors in the eel. BMC Developmental Biology 9(1): 71.

450 Davies WL, Hankins MW, Foster RG. 2010. Vertebrate ancient opsin and melanopsin:

451 divergent irradiance detectors. Photochemical \& Photobiological Sciences 9(11):1444-1457.

452 Doyle SE, Yoshikawa T, Hillson H, Menaker M. 2008. Retinal pathways influence temporal

453 niche. Proceedings of the National Academy of Sciences 105(35):13133-13138.

454 Falcón J. 1999. Cellular circadian clocks in the pineal. Progress in neurobiology 58(2):121-162.

455 García-Fernández JM, Cernuda-Cernuda R, Davies WI, Rodgers J, Turton M, Peirson SN,

456 Follett BK, Halford S, Hughes S, Hankins MW, Foster RG. 2015. The hypothalamic

457 photoreceptors regulating seasonal reproduction in birds: A prime role for VA opsin.

$458 \quad$ Frontiers in Neuroendocrinology 37: 13-28.

459 Grabherr MG, Haas BJ, Yassour M, Levin JZ, Thompson DA, Amit I, Adiconis X, Fan L,

460 Raychowdhury R, Zeng Q, Chen Z, Mauceli E, Hacohen N, Gnirke A, Rhind N, Palma

461 FD, Birren BW, Nusbaum C, Lindblad-Toh K, Friedman N, Regev A. 2011. Full-length 
462 463

464 465 466 467 468 469 470

transcriptome assembly from RNA-Seq data without a reference genome. Nature biotechnology 29(7):644-52.

Grone BP, Zhao S, Chen CC, Fernald RD. 2007. Localization and Diurnal Expression of Melanopsin, Vertebrate Ancient Opsin, and Pituitary Adenylate Cyclase-Activating Peptide mRNA in a Teleost Retina. Journal of biological rhythms 22(6): 558-561.

Hastings M, Maywood ES. 2000. Circadian clocks in the mammalian brain. Bioessays 22: 2331.

Kim D, Pertea G, Trapnell C, Pimentel H, Kelley R, Salzberg SL. 2013. TopHat2 accurate alignment of transcriptomes in the presence of insertions, deletions and gene fusions. Genome Biol 14:R36.

Kojima D, Fukada Y. 1999. Non-visual photoreception by a variety of vertebrate opsins. Rhodopsin and Phototransduction 265-282.

Kojima D, Mano H, Fukada Y. 2000. Vertebrate ancient-long opsin: a green-sensitive photoreceptive molecule present in zebrafish deep brain and retinal horizontal cells. Journal of Neuroscience 20: 2845-2851.

Langmead B, Salzberg SL. 2012. Fast gapped-read alignment with Bowtie 2. Nature Methods 9:357-359. DOI: 10.1038/nmeth.1923.

Li W, Godzik A. 2006. Cd-hit: A fast program for clustering and comparing large sets of protein or nucleotide sequences. Bioinformatics 22:1658-1659. DOI: 10.1093/bioinformatics/btl158.

Lin JJ, Wang FY, Li WH, Wang TY. 2017. The rises and falls of opsin genes in 59 ray-finned fish genomes and their implications for environmental adaptation. Scientific reports 7: 15568. 
484 Mas-Riera J. 1991. Changes during growth in the retinal structure of three hake species,

485 Merluccius spp. (Teleostei: Gadiformes), in relation to their depth distribution and feeding. 486 Journal of Experimental Marine Biology and Ecology 152(1): 91-104.

487 Masuda T, Iigo M, Mizusawa K, Aida K. 2003. Retina-type rhodopsin gene expressed in the 488 brain of a teleost, ayu (Plecoglossus altivelis). Zoological science 20: 989-997.

489 Minamoto T, Shimizu I. 2003. Molecular cloning and characterization of rhodopsin in a teleost 490 (Plecoglossus altivelis, Osmeridae). Comparative Biochemistry and Physiology Part B: $491 \quad$ Biochemistry and Molecular Biology 134: 559-570.

492 Moutsaki P, Whitmore D, Bellingham J, Sakamoto K, David-Gray ZK, Foster RG. 2003. 493 Teleost multiple tissue (tmt) opsin: a candidate photopigment regulating the peripheral clocks 494 of zebrafish?. Molecular brain research 112(1-2): 135-145.

Nariai N, Kojima K, Mimori T, Sato Y, Kawai Y, Yamaguchi-Kabata Y, Nagasaki M. 2014. TIGAR2: Sensitive and accurate estimation of transcript isoform expression with longer RNA-Seq reads. BMC Genomics 15:S5. DOI: 10.1186/1471-2164-15-S10-S5.

Okano T, Yoshizawa T, Fukada Y. 1994. Pinopsin is a chicken pineal photoreceptive molecule. Nature 372: 94-97.

Pankhurst NW, Conroy AM. 1987. Seasonal changes in reproductive condition and plasma levels of sex steroids in the blue cod, Parapercis colias (Bloch and Schneider)

502 (Mugiloididae). Fish Physiology and Biochemistry 4(1): 15-26.

Peirson SN, Halford S, Foster RG. 2009. The evolution of irradiance detection: melanopsin 504 and the non-visual opsins. Philosophical Transactions of the Royal Society B: Biological 505 Sciences 364: 2849-2865. 
506

507

508

509

510

511

512

513

514

515

516

517

518

519

520

521

522

523

524

525

526

527

528

Philp AR, Garcia-Fernandez JM, Soni BG, Lucas RJ, Bellingham J, Foster RG. 2000.

Vertebrate ancient (VA) opsin and extraretinal photoreception in the Atlantic salmon (Salmo salar). Journal of Experimental Biology 203: 1925-1936.

Pugh Jr EN, Lamb TD. 2000. Phototransduction in vertebrate rods and cones: Molecular mechanisms of amplification, recovery and light adaptation. Handbook of Biological Physics 3: $3183-255$.

Sakata R, Kabutomori R, Okano K, Mitwui H, Takemura A, Miwa T, Yamamoto H, Okano T. 2015. Rhodopsin in the dark hot sea: molecular analysis of rhodopsin in a Snailfish, Careproctus rhodomelas, living near the deep-sea hydrothermal vent. Plos ONE 10(8): e0135888 Doi:10.1371/journal.pone.0135888.

Schmieder R, Edwards R. 2011. Quality control and preprocessing of metagenomic datasets. Bioinformatics 27:863-864. DOI: 10.1093/bioinformatics/btr026.

Shand J, Hart NS, Thomas N, Partridge JC. 2002. Developmental changes in the cone visual pigments of black bream Acanthopagrus butcheri. Journal of Experimental Biology 205: $3661-3667$

Smith-Unna R, Boursnell C, Patro R, Hibberd JM, Kelly S. 2015. TransRate: reference free quality assessment of de-novo transcriptome assemblies. Genome research 26(8): 1134-1144.

Soni BG, Foster RG. 1997. A novel and ancient vertebrate opsin. FEBS letters 406: 279-283.

Stamatakis A. 2014. RAxML version 8: A tool for phylogenetic analysis and post-analysis of large phylogenies. Bioinformatics 30:1312-1313. DOI: 10.1093/bioinformatics/btu033.

Stanke M, Morgenstern B. 2005. AUGUSTUS: a web server for gene prediction in eukaryotes that allows user-defined constraints. Nucleic acids research 33(Web Server issue): W465W467.

Peer) reviewing PDF | (2019:04:37146:2:0:NEW 18 Nov 2019) 
529 Suzuki K. 1975. The significance of photoperiod on the gonadal development of ayu

530 Plecoglossus altivelis. K Suzuki Doctral dissertation, University of Tokyo: Tokyo.

531 Tada T, Altun A, Yokoyama S. 2009. Evolutionary replacement of UV vision by violet vision 532 in fish Proceedings of the National Academy of Sciences 106(41): 17457-17462.

533 Tatsukawa K. 2003. Eel resources in East Asia. Eel Biology 293-298.

534 Terakita A, Kawano-Yamashita E, Koyanagi M. 2012. Evolution and diversity of opsins. 535 Wiley Interdisciplinary Reviews: Membrane Transport and Signaling 1(1):104-111.

536 Terakita A. 2005. The opsins. Genome biology 6: 213.

537 Tsukamoto K. 1992. Discovery of the spawning area for Japanese eel. Nature 356:789-791.

538 Van Gelder RN. 2001. Non-visual ocular photoreception. Ophthalmic Genetics 22(4): 195-205.

539 Wang FY, Fu WC, Wang IL, Yan HY, Wang TY. 2014. The giant Mottled Eel, Anguilla 540 marmorata, used blue-shifted rod photoreceptors during upstream migration. Plos ONE 9(8): 541 e103953 Doi:10.1371/journal.pone.0103953.

542 Yau KW, Hardie RC. Phototransduction motifs and variations. 2009. Cell 139(2): 246-264.

543 Zhang H, Futami K, Horie N, Okamura A, Utoh T, Mikawa N, Yamada Y, Tanaka S,

544 Okamoto N. 2000. Molecular cloning of fresh water and deep-sea rod opsin genes from 545 Japanese eel Anguilla japonica and expressional analyses during sexual maturation. FEBS $546 \quad$ letters 469(1): 39-43.

547 Zhang H, Futami K, Yamada Y, Horie N, Okamura A, Utoh T, Mikawa N, Tanaka S,

548 Okamoto N, Oka HP. 2002. Isolation of freshwater and deep-sea type opsin genes from the 549 common Japanese conger. Journal of Fish Biology 61: 313-324. 


\section{Figure captions}

551 Fig. 1. Flowchart of the present study.

552

553 Fig. 2. Diagram showing the dorsal view (a) and sagittal plane (b) of the eel brain. Ob, 554 olfactory bulb; Tel, telencephalon; TeO, optic tectum; Cb, cerebellum; Mo, medulla oblongata;

555 P, pineal gland; SD, saccus dorsalis; PON, preoptic nucleus; SV, saccus vasculosus.

556

557

558

559

560

561

562

563

564

565

566

567

568

569

570

571

572

Fig. 3. Microphotographs of histological sections of different stages of eel testis and changes of gonadosomatic index (GSI) after hormonally induced sexual maturation. (A) immature testis, (B) mature testis, (C) GSI. Scale bar $=200 \mu \mathrm{m}$.

\section{Fig. 4. Phylogeny of vertebrate visual and non-visual opsins. One thousand bootstrap} repetitions were performed and values are shown at the inner nodes. The zebrafish beta 1 adrenergic receptor was used as an outgroup to root the tree. Analysis was performed with multiple alignments from the amino acid sequence by using ClustalW program. Bold is indicated the visual and non-visual opsin families of Japanese eel, A. japonica.

\section{Fig. 5. Visual opsin mRNA level in the retina of sexually immature and mature male}

Japanese eel. For the artificially induced sexual maturation, hCG was intraperitoneally injected to the experimental fish group $(\mathrm{n}=6)$ at a concentration of $1 \mathrm{IU} / \mathrm{g}$ body weight. Immature fish was sampled before hCG injection $(n=6)$. Eight weeks after injection, retina was sampled and used for total RNA extraction and cDNA synthesis. The mRNA expression of visual opsin in each sample was measured real-time qPCR. Boxplots show min and max values (whiskers), first and 
573 third quartiles (box limits), and median (box inner line) of mRNA levels. The asterisk above

574 each bar indicates significant differences according to the Unparied t test $\left({ }^{*} P<0.05\right.$, ${ }^{* *} P<$ $5750.01)$.

576

577 Fig. 6. Non-visual opsin mRNA level in the retina of sexually immature and mature male

578 Japanese eel. For the artificially induced sexual maturation, hCG was intraperitoneally injected 579 to the experimental fish group $(\mathrm{n}=6)$ at a concentration of $1 \mathrm{IU} / \mathrm{g}$ body weight. Immature fish was 580 sampled before $\mathrm{hCG}$ injection $(\mathrm{n}=6)$. Eight weeks after injection, retina was sampled and used 581 for total RNA extraction and cDNA synthesis. The mRNA expression of non-visual opsin in 582 each sample was measured real-time qPCR. Boxplots show min and max values (whiskers), first 583 and third quartiles (box limits), and median (box inner line) of mRNA levels. The asterisk above 584 each bar indicates significant differences according to the Unparied t test $\left(* P<0.05,{ }^{* *} P<\right.$ $5850.01)$.

586

587 Fig. 7. Visual opsin mRNA level in the brain of sexually immature and mature male

588 Japanese eel. For the artificially induced sexual maturation, hCG was intraperitoneally injected 589 to the experimental fish group $(n=6)$ at a concentration of $1 \mathrm{IU} / \mathrm{g}$ body weight. Immature fish was 590 sampled before hCG injection $(\mathrm{n}=6)$. Eight weeks after injection, brain was sampled and used for 591 total RNA extraction and cDNA synthesis. The mRNA expression of visual opsin in each sample

592 was measured real-time qPCR. Boxplots show min and max values (whiskers), first and third 593 quartiles (box limits), and median (box inner line) of mRNA levels. The asterisk above each bar 594 indicates significant differences according to the Unparied t test $\left({ }^{*} P<0.05,{ }^{*} P<0.01\right)$. 
596 Fig. 8. Non-visual opsin mRNA level in the brain of sexually immature and mature male

597 Japanese eel male. Boxplots show min and max values (whiskers), first and third quartiles (box

598 limits), and median (box inner line) of mRNA levels. For the artificially induced sexual

599 maturation, hCG was intraperitoneally injected to the experimental fish group $(\mathrm{n}=6)$ at a

600 concentration of $1 \mathrm{IU} / \mathrm{g}$ body weight. Immature fish was sampled before hCG injection $(\mathrm{n}=6)$.

601 Eight weeks after injection, brain was sampled and used for total RNA extraction and cDNA

602 synthesis. The mRNA expression of non-visual opsin in each sample was measured real-time

603 qPCR. Boxplots show min and max values (whiskers), first and third quartiles (box limits), and

604 median (box inner line) of mRNA levels. The asterisk above each bar indicate significant

605 differences according to the Unparied t test $(* P<0.05, * * P<0.01)$.

606

607 Fig. 9. Non-visual opsin mRNA level in the brain of sexually immature and mature male

608 Japanese eel male. Boxplots show min and max values (whiskers), first and third quartiles (box

609 limits), and median (box inner line) of mRNA levels. For the artificially induced sexual

610 maturation, hCG was intraperitoneally injected to the experimental fish group $(n=6)$ at a

611 concentration of $1 \mathrm{IU} / \mathrm{g}$ body weight. Immature fish was sampled before hCG injection $(\mathrm{n}=6)$.

612 Eight weeks after injection, brain was sampled and used for total RNA extraction and cDNA

613 synthesis. The mRNA expression of non-visual opsin in each sample was measured real-time

614 qPCR. Boxplots show min and max values (whiskers), first and third quartiles (box limits), and

615 median (box inner line) of mRNA levels. The asterisk above each bar indicate significant

616 differences according to the Unparied t test $(* P<0.05, * * P<0.01)$.

617 
Figure 1

Flowchart of the present study.

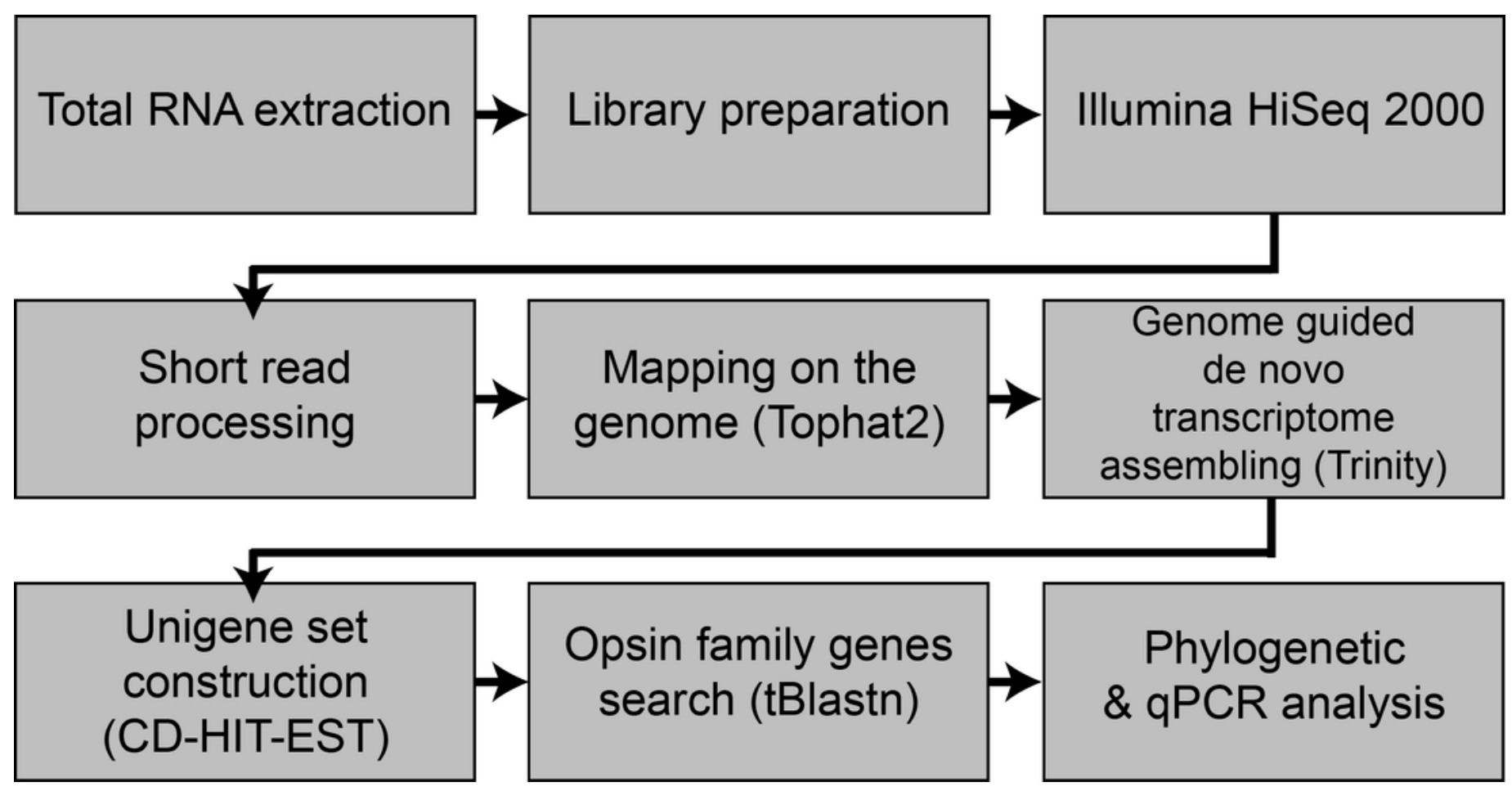


Figure 2

Diagram showing the dorsal view (a) and sagittal plane (b) of the eel brain.

$\mathrm{Ob}$, olfactory bulb; Tel, telencephalon; $\mathrm{TeO}$, optic tectum; $\mathrm{Cb}$, cerebellum; Mo, medulla oblongata; P, pineal gland; SD, saccus dorsalis; PON, preoptic nucleus; SV, saccus vasculosus.

A

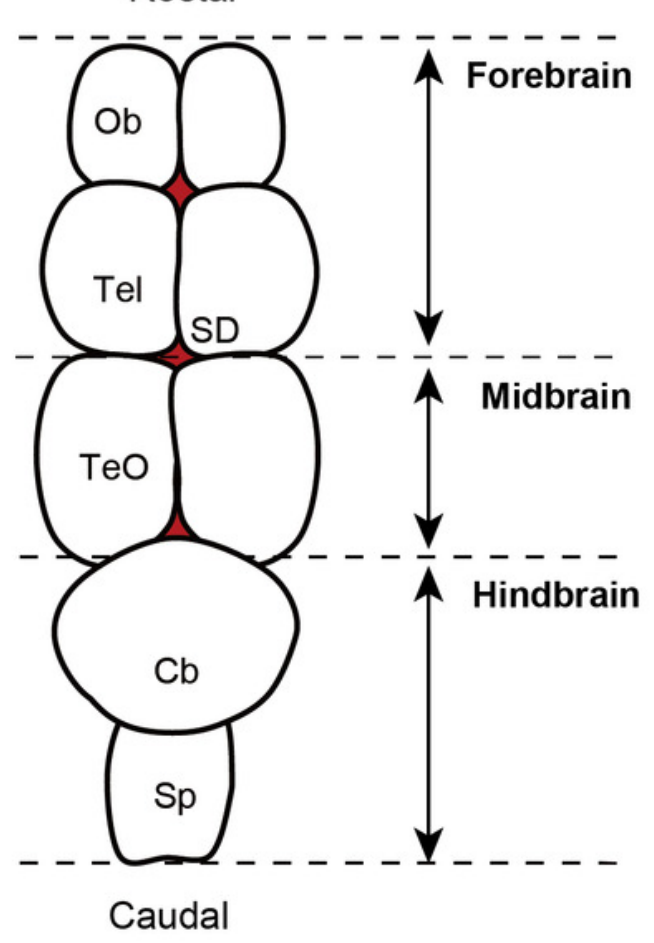

B

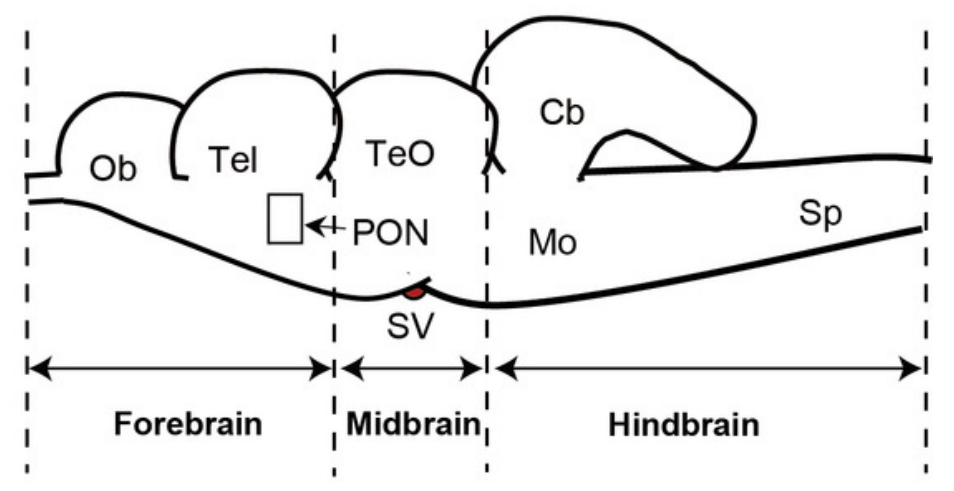

Fore-brain (prosencephalon): olfactory bulb, telencephalon, diencephalon Mid-brain (Mesencephalon): optictectum, saccus vasculosus Hind-brain (Rhombencephalon): cerebellum, medulla oblongata, spinal cord 


\section{Figure 3}

Microphotographs of histological sections of different stages of eel testis and changes of gonadosomatic index (GSI) after hormonally induced sexual maturation.

(A) immature testis, (B) mature testis, (C) GSI. Scale bar $=200 \mu \mathrm{m}$.
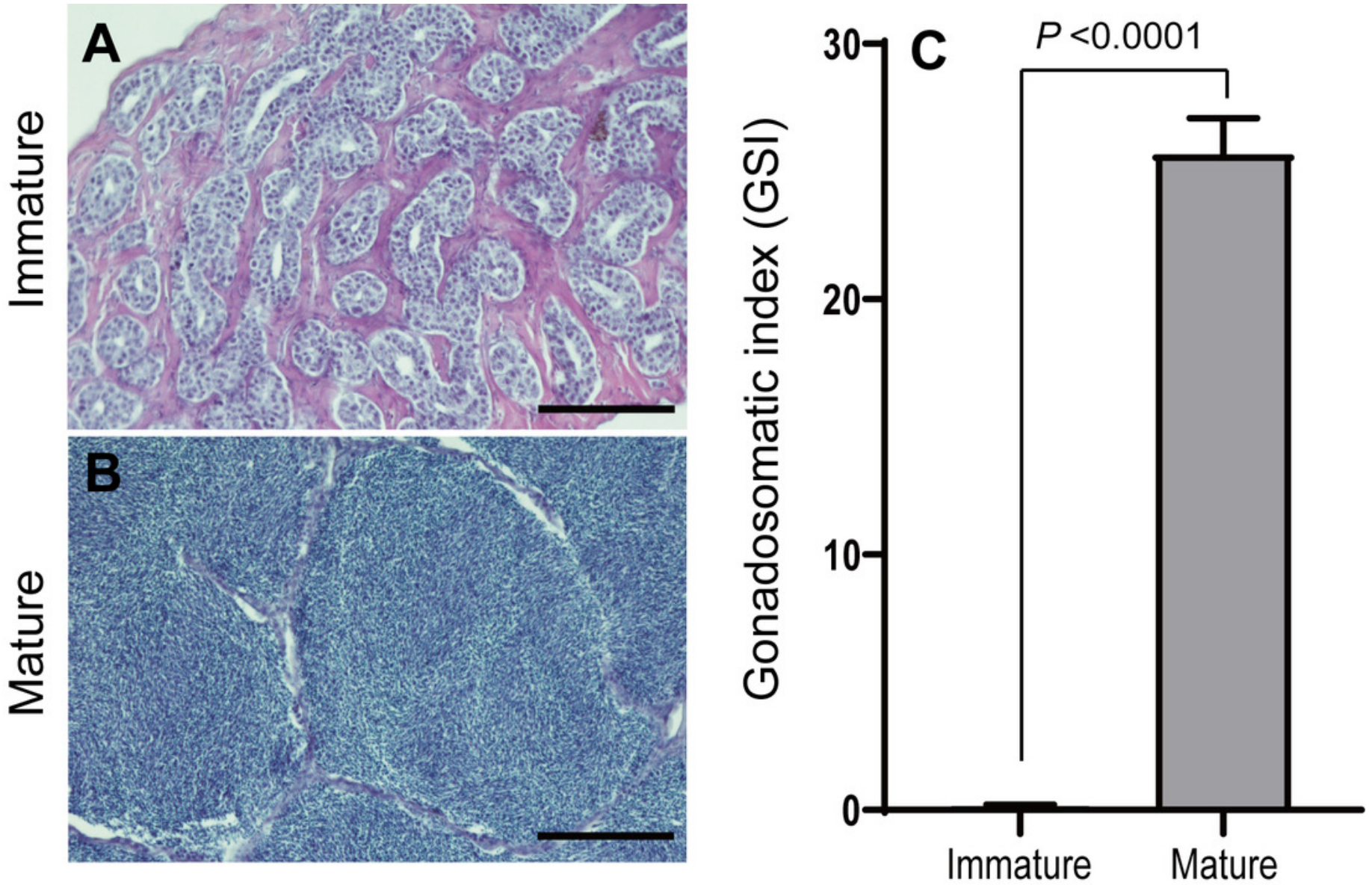
Figure 4

Phylogeny of vertebrate visual and non-visual opsins. One thousand bootstrap repetitions were performed and values are shown at the inner nodes.

The zebrafish beta 1 adrenergic receptor was used as an outgroup to root the tree. Analysis was performed with multiple alignments from the amino acid sequence by using ClustalW program. Bold is indicated the visual and non-visual opsin families of Japanese eel, $A$. japonica.

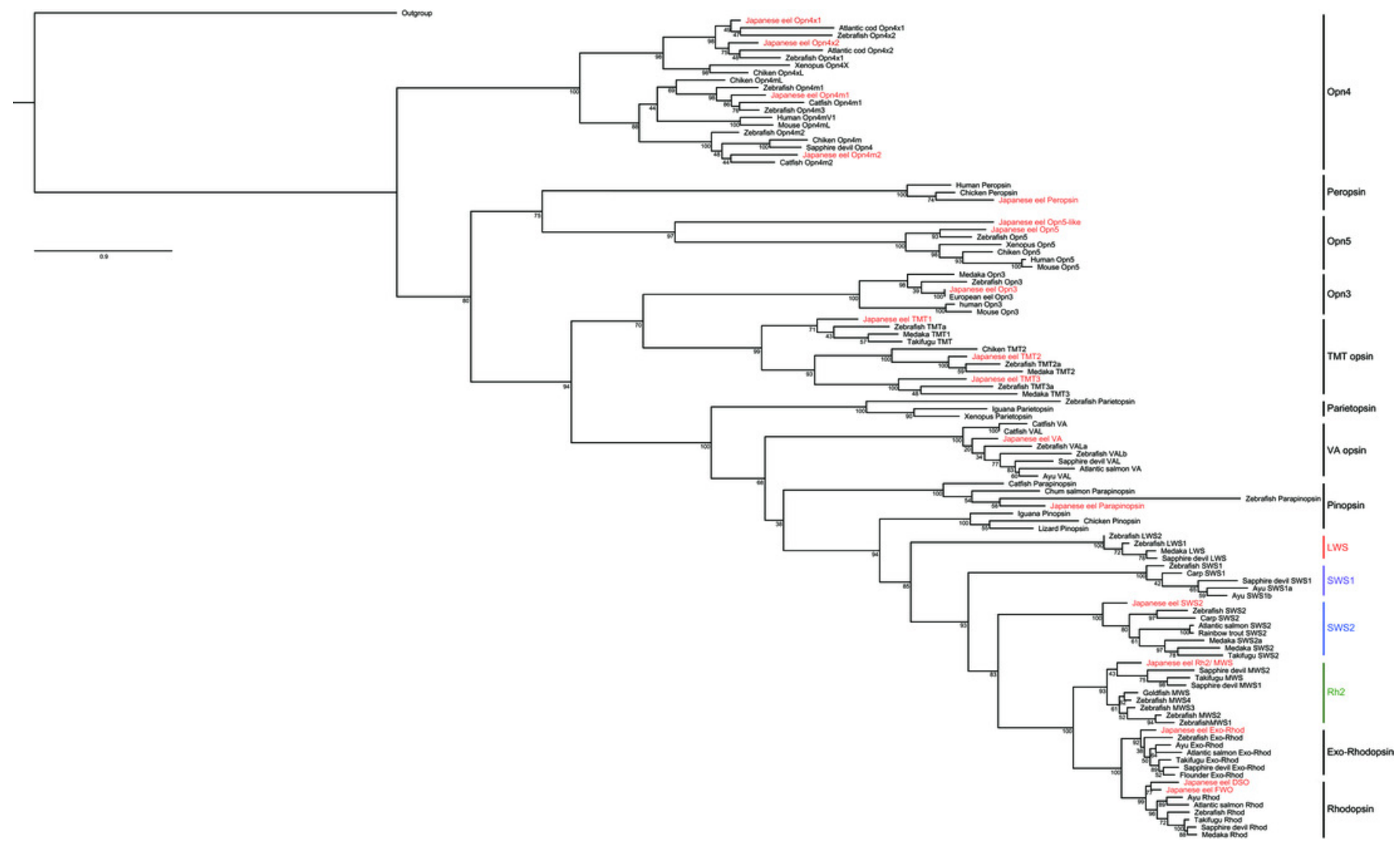




\section{Figure 5}

Visual opsin mRNA level in the retina of sexually immature and mature male Japanese eel.

For the artificially induced sexual maturation, hCG was intraperitoneally injected to the experimental fish group $(n=6)$ at a concentration of $1 \mathrm{lU} / \mathrm{g}$ body weight. Immature fish was sampled before hCG injection $(n=6)$. Eight weeks after injection, retina was sampled and used for total RNA extraction and cDNA synthesis. The mRNA expression of visual opsin in each sample was measured real-time qPCR. Boxplots show min and max values (whiskers), first and third quartiles (box limits), and median (box inner line) of mRNA levels. The asterisk above each bar indicates significant differences according to the Unparied test $(* P<0.05$, $* * P<0.01)$.

\section{Visual opsin mRNA in retina}
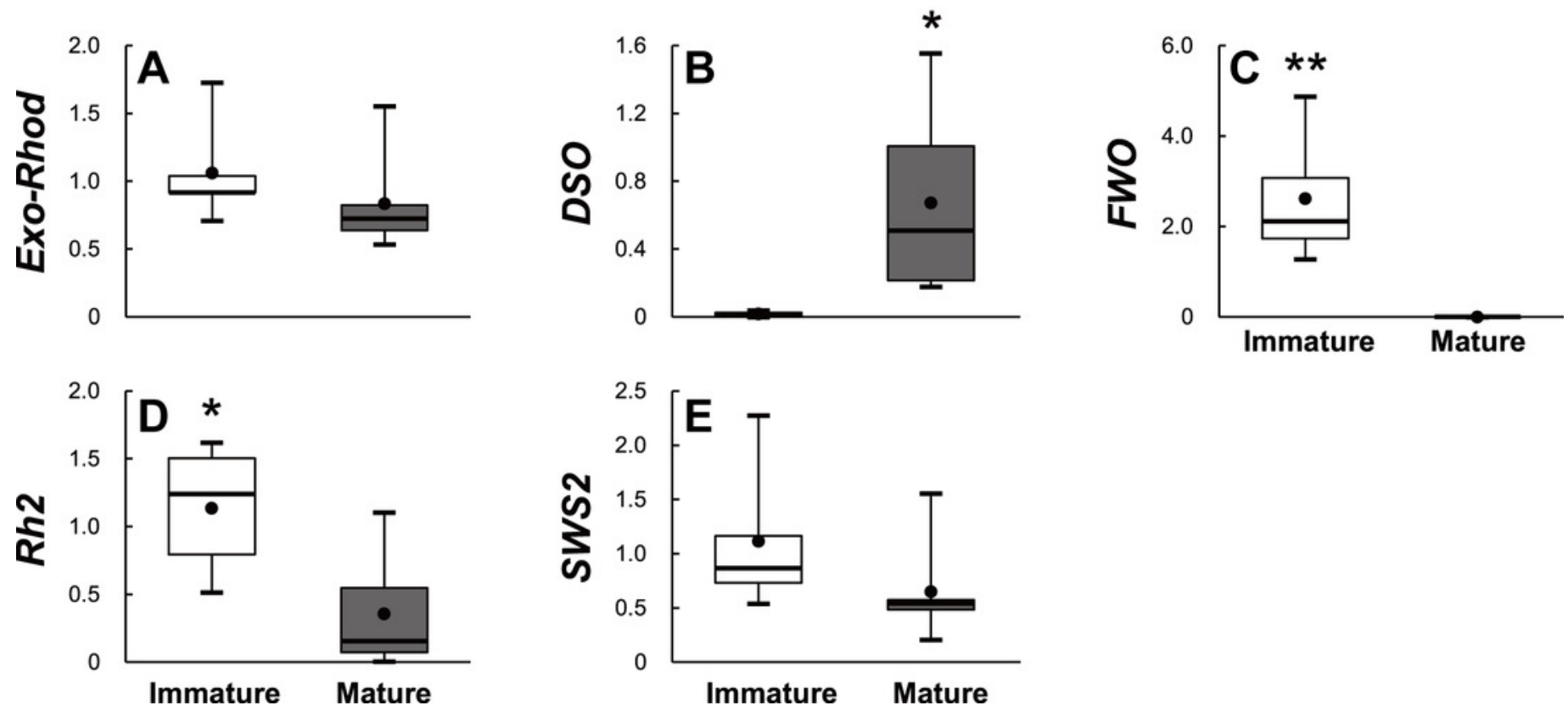


\section{Figure 6}

Non-visual opsin mRNA level in the retina of sexually immature and mature male Japanese eel.

For the artificially induced sexual maturation, hCG was intraperitoneally injected to the experimental fish group $(n=6)$ at a concentration of $1 \mathrm{IU} / \mathrm{g}$ body weight. Immature fish was sampled before $h C G$ injection $(n=6)$. Eight weeks after injection, retina was sampled and used for total RNA extraction and CDNA synthesis. The mRNA expression of non-visual opsin in each sample was measured real-time qPCR. Boxplots show min and max values (whiskers), first and third quartiles (box limits), and median (box inner line) of mRNA levels. The asterisk above each bar indicates significant differences according to the Unparied t test $(* P<0.05$, $* * P<0.01)$. 


\section{Non-visual opsin mRNA in retina}
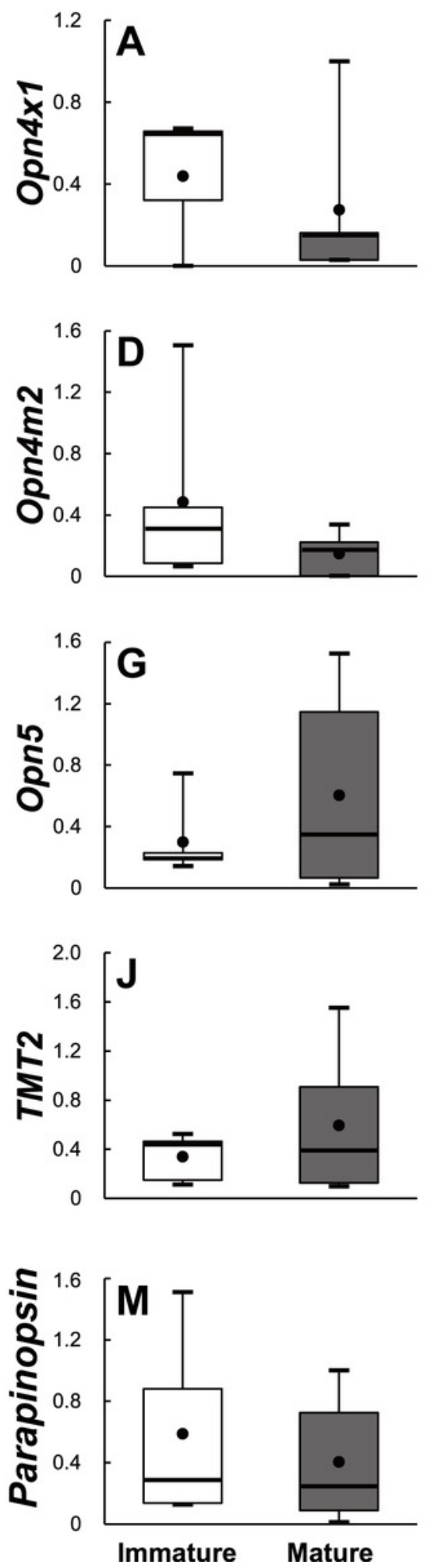
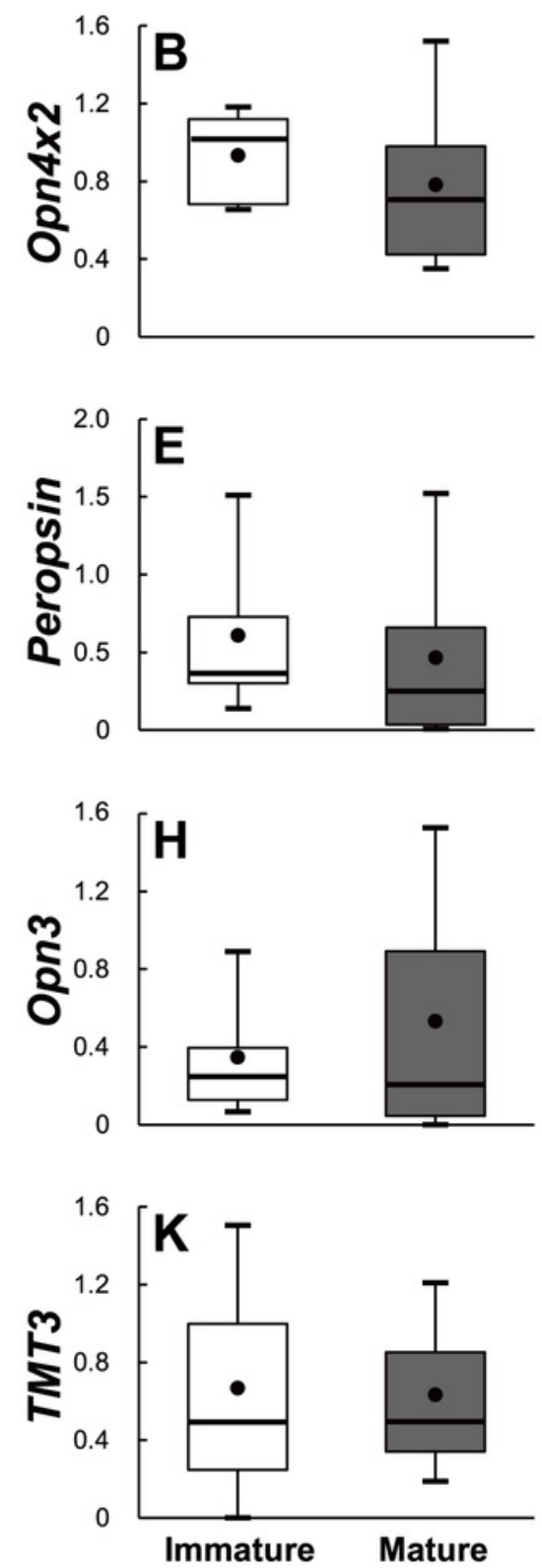
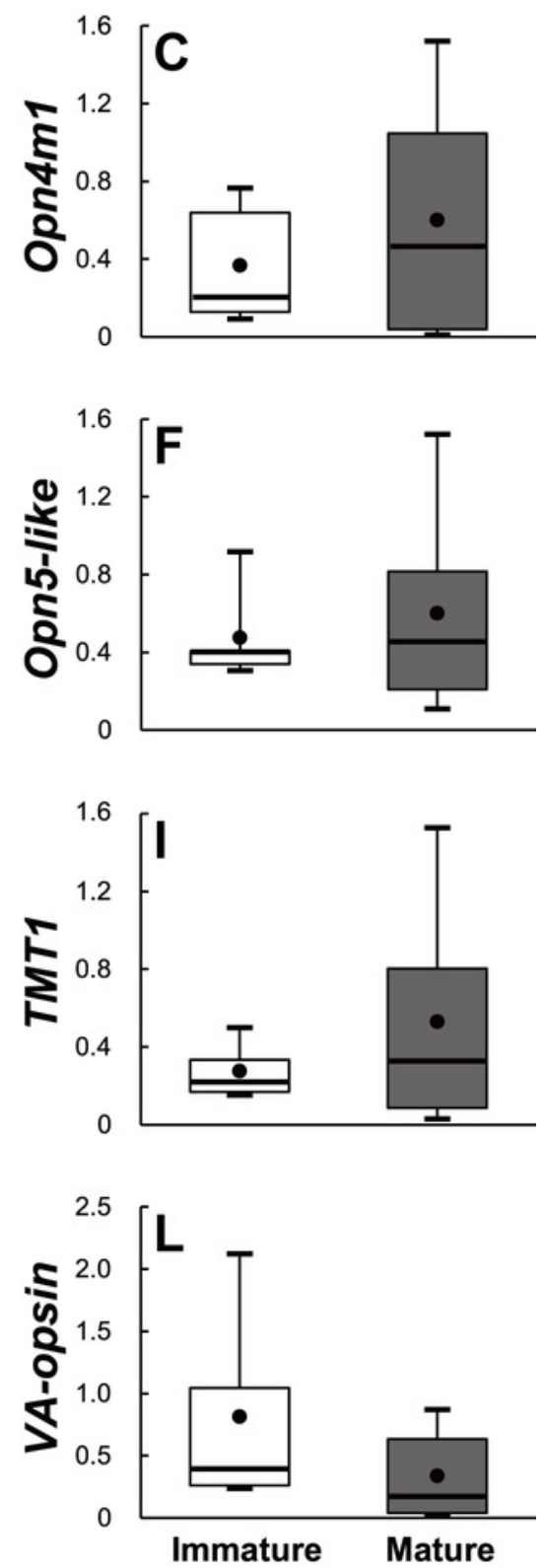


\section{Figure 7}

Visual opsin mRNA level in the brain of sexually immature and mature male Japanese eel.

For the artificially induced sexual maturation, hCG was intraperitoneally injected to the experimental fish group $(n=6)$ at a concentration of $1 \mathrm{lU} / \mathrm{g}$ body weight. Immature fish was sampled before $\mathrm{hCG}$ injection $(n=6)$. Eight weeks after injection, brain was sampled and used for total RNA extraction and cDNA synthesis. The mRNA expression of visual opsin in each sample was measured real-time qPCR. Boxplots show min and max values (whiskers), first and third quartiles (box limits), and median (box inner line) of mRNA levels. The asterisk above each bar indicates significant differences according to the Unparied t test $(* P<0.05$, $* * P<0.01)$. 


\section{Visual opsin mRNA in brain}
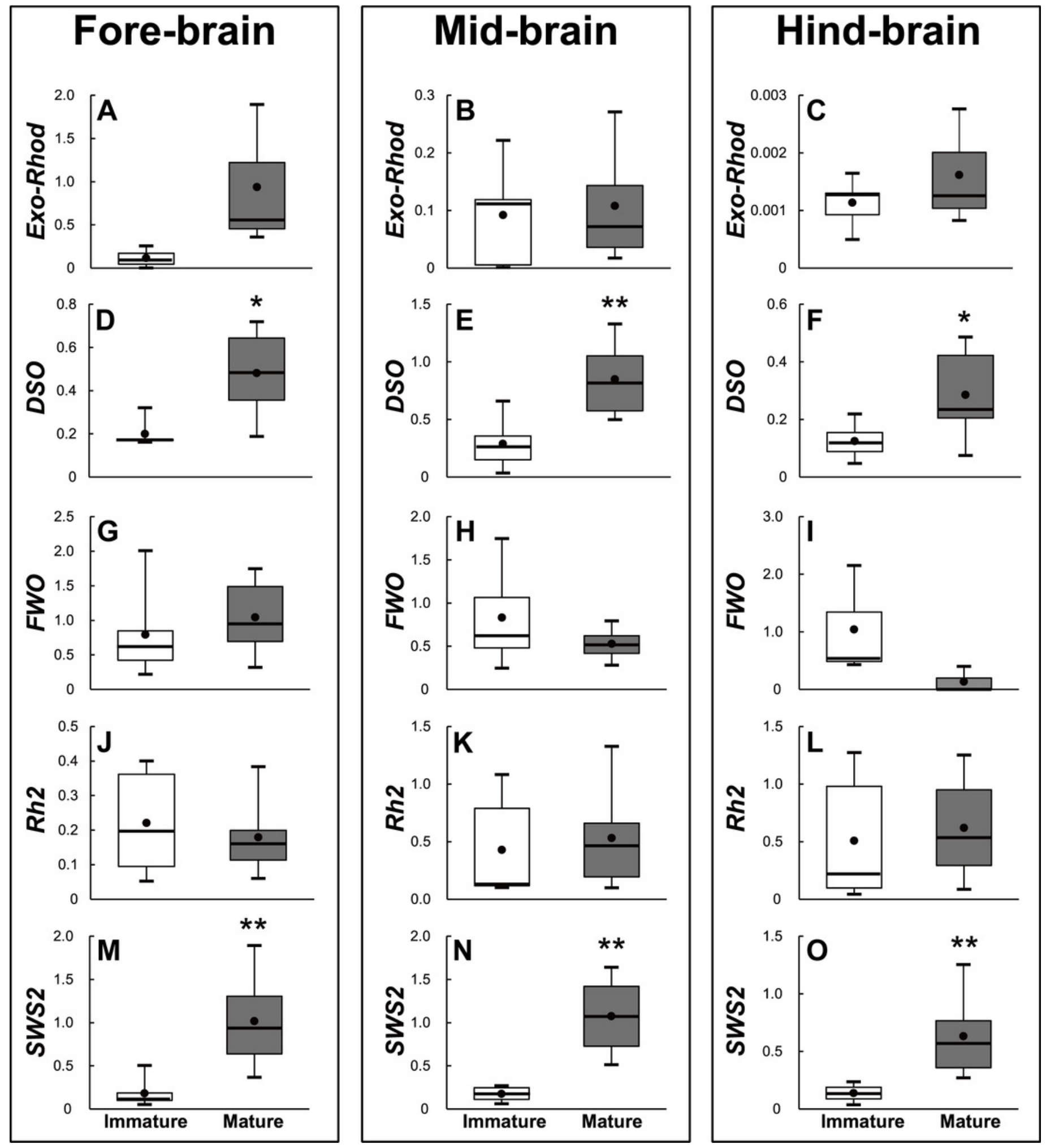


\section{Figure 8}

Non-visual opsin mRNA level in the brain of sexually immature and mature male Japanese eel male.

Boxplots show min and max values (whiskers), first and third quartiles (box limits), and median (box inner line) of mRNA levels. For the artificially induced sexual maturation, hCG was intraperitoneally injected to the experimental fish group $(n=6)$ at a concentration of 1 IU/g body weight. Immature fish was sampled before hCG injection $(n=6)$. Eight weeks after injection, brain was sampled and used for total RNA extraction and CDNA synthesis. The mRNA expression of non-visual opsin in each sample was measured real-time qPCR. Boxplots show min and max values (whiskers), first and third quartiles (box limits), and median (box inner line) of mRNA levels. The asterisk above each bar indicate significant differences according to the Unparied t test $(* P<0.05, * * P<0.01)$. 
Non-visual opsin mRNA in brain
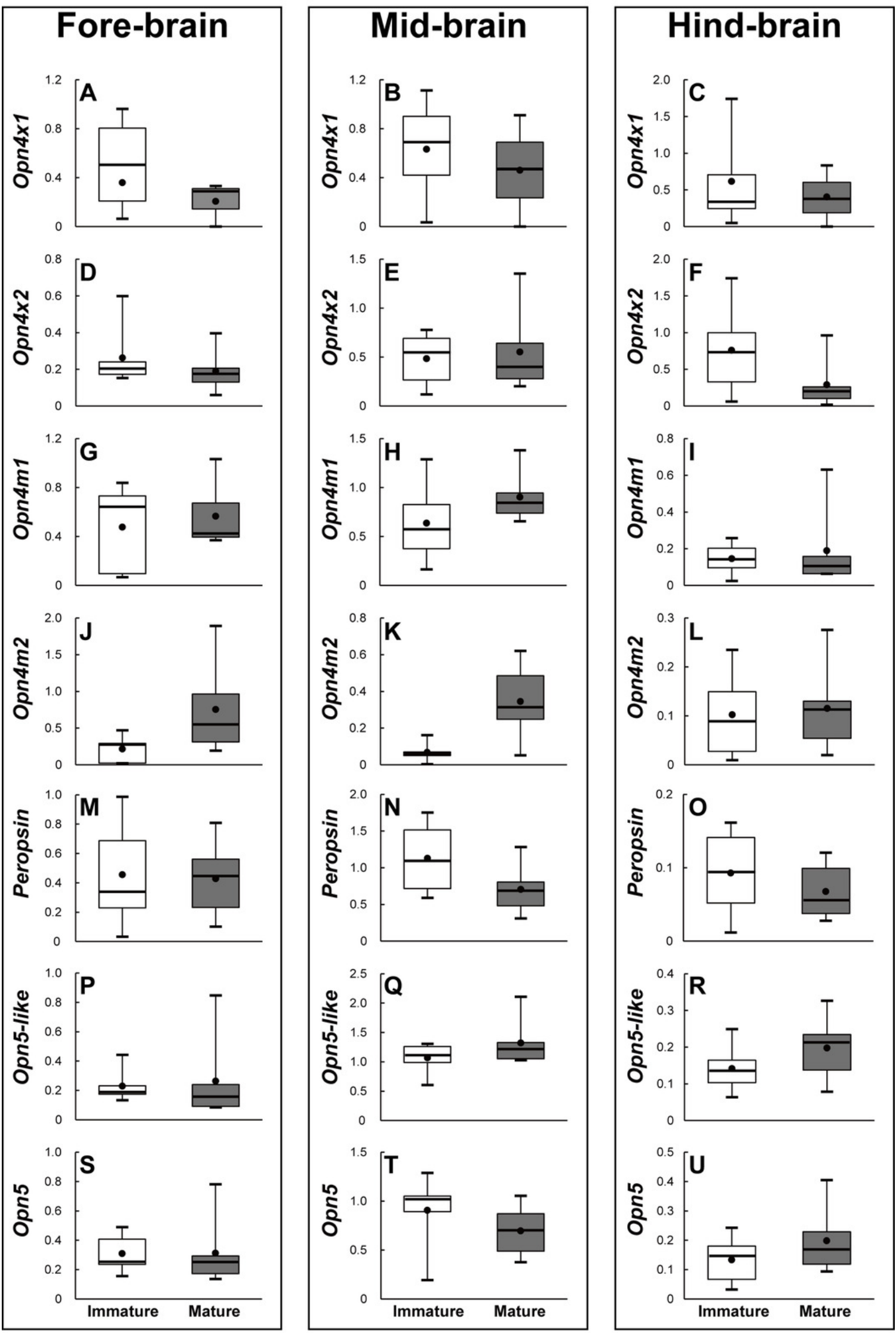


\section{Figure 9}

Non-visual opsin mRNA level in the brain of sexually immature and mature male Japanese eel male.

Boxplots show min and max values (whiskers), first and third quartiles (box limits), and median (box inner line) of mRNA levels. For the artificially induced sexual maturation, hCG was intraperitoneally injected to the experimental fish group $(n=6)$ at a concentration of 1 IU/g body weight. Immature fish was sampled before hCG injection $(n=6)$. Eight weeks after injection, brain was sampled and used for total RNA extraction and CDNA synthesis. The mRNA expression of non-visual opsin in each sample was measured real-time qPCR. Boxplots show min and max values (whiskers), first and third quartiles (box limits), and median (box inner line) of mRNA levels. The asterisk above each bar indicate significant differences according to the Unparied t test $(* P<0.05, * * P<0.01)$. 


\section{Non-visual opsin mRNA in brain}
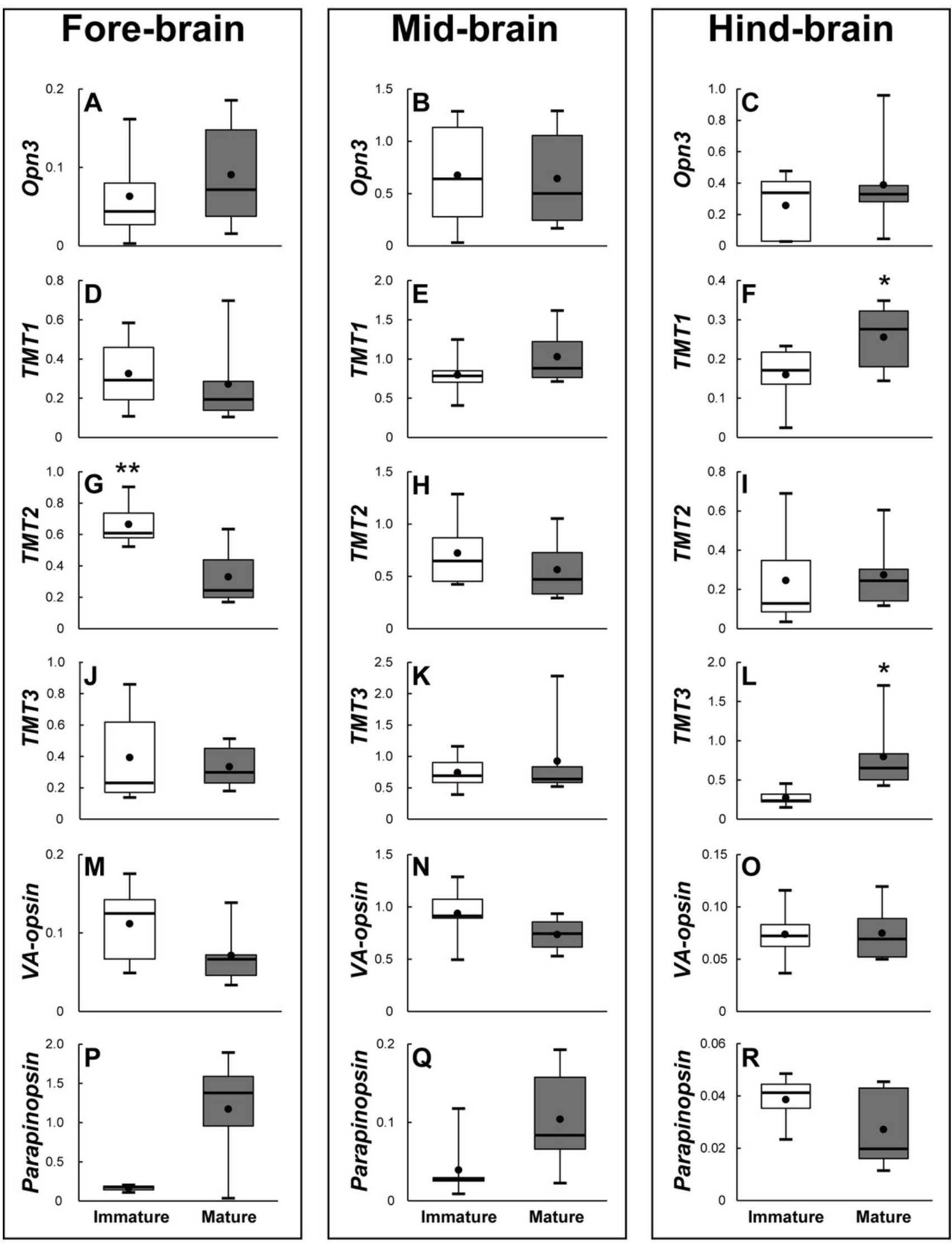